\title{
Aberrant right subclavian artery leading to prenatal diagnosis of Koolen de Vries syndrome
}

\author{
Parthenis Christos $(1)$, Panagopoulos Perikles \\ $3^{\text {rd }}$ Department of Obstetrics and Gynaecology, ATTIKON University Hospital Athens
}

\begin{abstract}
Koolen de Vries syndrome is a rare genetic disorder with an estimated prevalence 1:16000 in the general population but it is considered to be an underdiagnosed syndrome. We report a 17q21.31 microdeletion which was diagnosed prenatally in a fetus with aberrant right subclavian artery (ARSA). Koolen de Vries syndrome is a rare chromosomal abnormality and according to the literature this is the first case in which the detection of ARSA led to this syndrome.
\end{abstract}

Key words: ARSA; 17q21.31 microdeletion; Koolen de Vries

Ginekologia Polska 2021; 92, 8: 597-598

\section{INTRODUCTION}

Koolen de Vries syndrome is a chromosomal abnormality involving the long arm of chromosome 17. It is also known as 17q21.31 microdeletion syndrome. The phenotypes of this syndrome are highly variable, therefore a huge variety of abnormalities is reported including characteristic craniofacial malformations, heart abnormalities, skeletal anomalies and renal defects. We report a 17q21.31 microdeletion which was diagnosed prenatally in a fetus with aberrant right subclavian artery (ARSA). According to the literature this is the first case in which the detection of ARSA led to Koolen de Vries syndrome.

\section{CASE REPORT}

A 19-year-old woman presented in her first pregnancy to our Fetal Medicine Unit for a routine anomaly scan at 20 weeks of gestation. We performed a transabdominal ultrasound scan, and an aberrant right subclavian artery was revealed (Fig. 1.). The detection of ARSA was performed in the three vessels and trachea axial view. After a genetic counseling, an amniocentesis was performed. After the performed array CGH of the extracted DNA, a 17q21.31 deletion was found. The size of the deletion was $231 \mathrm{~Kb}$ and encompassed the KANSL1 gene. The haploinsufficiency of KANSL1 gene is considered adequate to cause the full Koolen de Vries phenotype.

\section{DISCUSSION}

Koolen de Vries syndrome is a rare genetic disorder with an estimated prevalence 1:16000 in the general population [1] but it is considered an underdiagnosed syndrome as the real genetic cause is often not detected in patients with developmental delay [2].

The deletion or microduplication of the 17q21.31 region was recognized only with the development of the array-CGH technique. The 17q21.31 region, typically encompassing at least six genes related to the phenotype, including CRHR1 (OMIM 122561), STH (OMIM 607067), MAPT (OMIM 157140), KIAA1267, C17orf69 and KANSL1 (0MIM 612452) [3, 4].

Koolen de Vries syndrome is associated with developmental delays. Craniofacial characteristics include long face, up slanting palpebral fissures, tubular nose with bulbous nasal tip, large prominent ears, thin upper and everted lower lip and long slender fingers [4].

Brain abnormalities are also present consisting of ventriculomegaly, corpus callosum defects, heterotopias and Arnold Chiari I malformation [4]. One of the most frequent deformity consisted of unilateral or bilateral hyperechogenic kidneys. Congenital heart conditions are also observed including atrial (ASD) and ventricular septal defects (VSD), pulmonary and mitral valve de- 


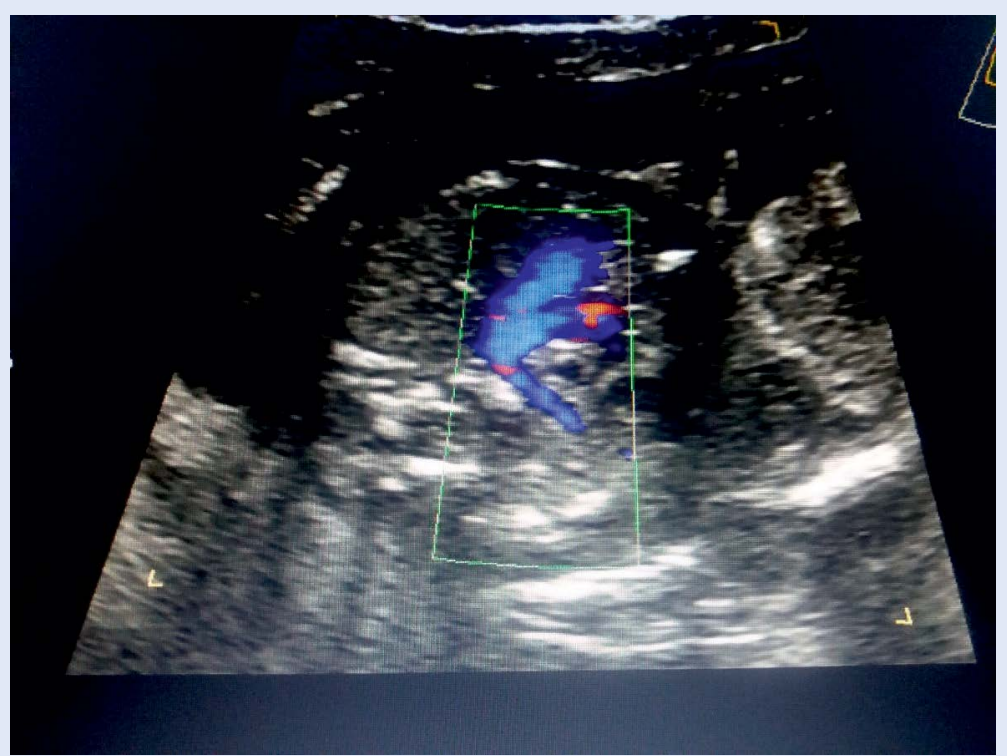

Figure 1. Aberrant right subclavian artery

formities, hypoplastic left heart syndrome (HLHS) and total anomalous pulmonary veins drainage [4]. To our knowledge we present the first case of Koolen DeVries syndrome after prenatal diagnosis of aberrant right subclavian artery.

ARSA is an abnormality in the branching pattern of aortic arch. When there is ARSA the right subclavian artery arises from the descending aorta, therefore we have four instead of three branches (right subclavian artery, left subclavian artery, left common carotid artery and right common carotid artery). ARSA is observed in $1.5 \%$ of normal individuals, $30 \%$ in fetuses with Down's Syndrome and 18\% in fetuses with trisomy 18 [5].

This case is an important addition to the existing knowledge about the 17q21.31 microdeletion syndrome.

\section{Conflict of interests}

The authors declare no conflict of interests.

\section{REFERENCES}

1. Shaw-Smith C, Pittman AM, Willatt L, et al. Microdeletion encompassing MAPT at chromosome 17q21.3 is associated with developmental delay and learning disability. Nat Genet. 2006; 38(9): 1032-1037, doi: 10.1038/ng1858, indexed in Pubmed: 16906163.

2. Koolen DA, Sharp AJ, Hurst JA, et al. Clinical and molecular delineation of the 17q21.31 microdeletion syndrome. J Med Genet. 2008; 45(11): 710-720, doi: 10.1136/jmg.2008.058701, indexed in Pubmed: 18628315.

3. Koolen DA, Pfundt R, Linda K, et al. DDD Study. The Koolen-de Vries syndrome: a phenotypic comparison of patients with a $17 q 21.31$ microdeletion versus a KANSL1 sequence variant. Eur J Hum Genet. 2016; 24(5): 652-659, doi: 10.1038/ejhg.2015.178, indexed in Pubmed: 26306646.

4. Dias J, Van Nguyen N, Georgiev P, et al. Structural analysis of the KANSL1/WDR5/KANSL2 complex reveals that WDR5 is required for efficient assembly and chromatin targeting of the NSL complex. Genes Dev. 2014; 28(9): 929-942, doi: 10.1101/gad.240200.114, indexed in Pubmed: 24788516.

5. Agathokleous M, Chaveeva P, Poon LCY, et al. Meta-analysis of second-trimester markers for trisomy 21. Ultrasound Obstet Gynecol. 2013; 41(3): 247-261, doi: 10.1002/uog.12364, indexed in Pubmed: 23208748. 